\title{
Cylindrical Projection of Electrostatic Potential and Image Analysis Tools for Damaged DNA. The Substitution of Thymine with Thymine Glycol
}

\author{
Maciej Haranczyk, Giovanni Lupica, Iwona Dąbkowska, Maciej Gutowski \\ Supporting information
}

Color versions of figures presented in the paper:

Figure 1. Thymine and atom numbering.

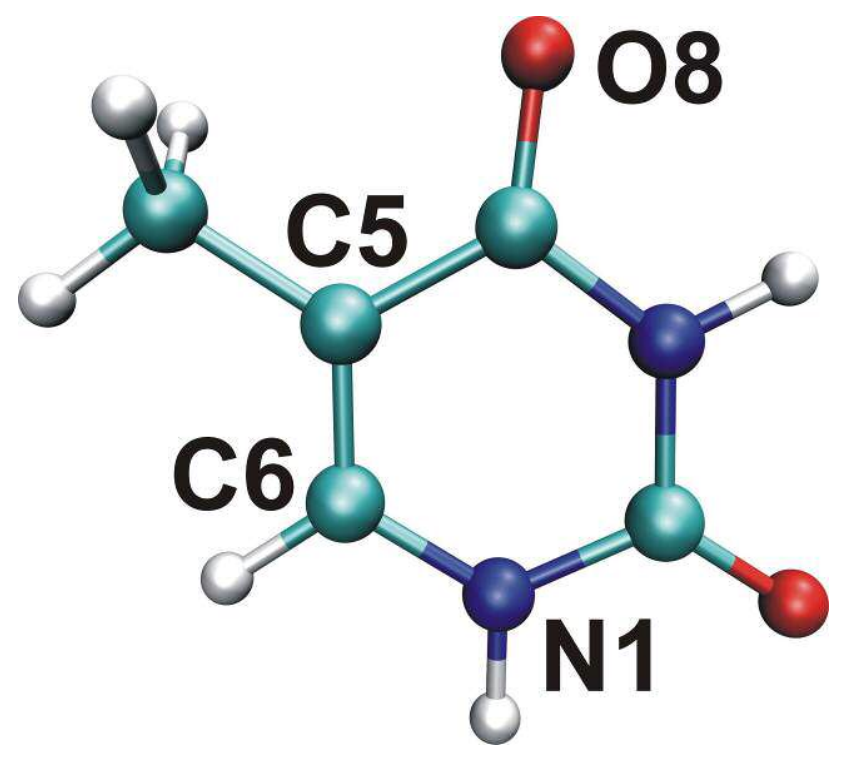


Figure 2. Three rotamers of thymine glycol.
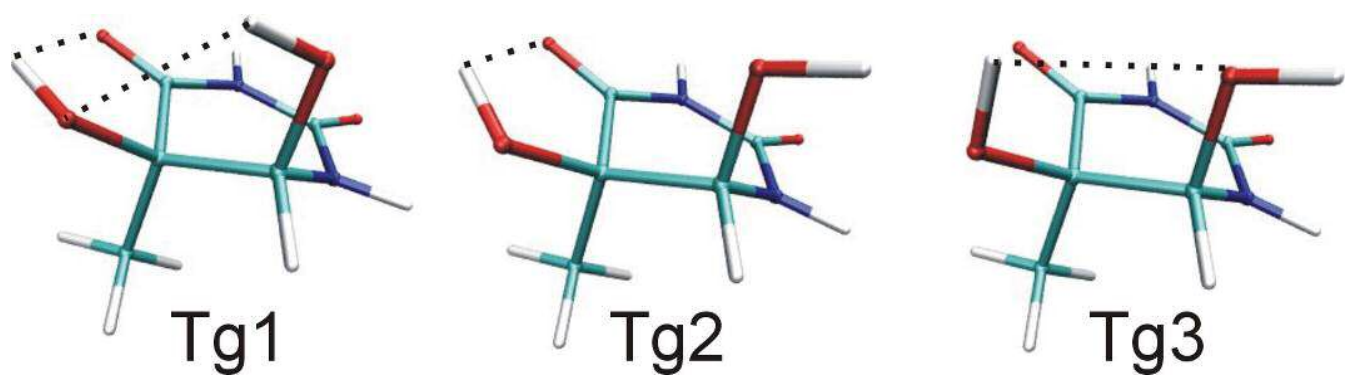
Figure 3. Three rotamers of thymine glycol connected to a sugar unit.

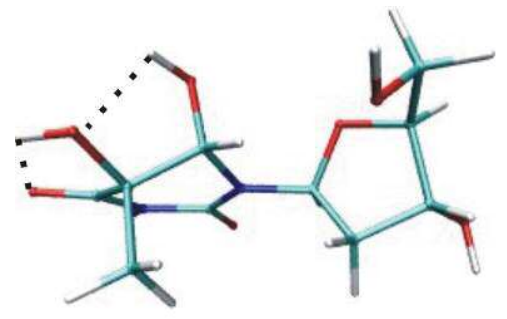

sTg1

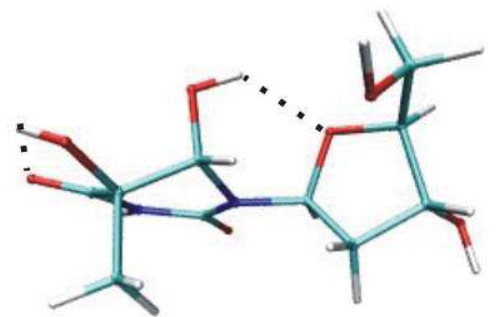

sTg2

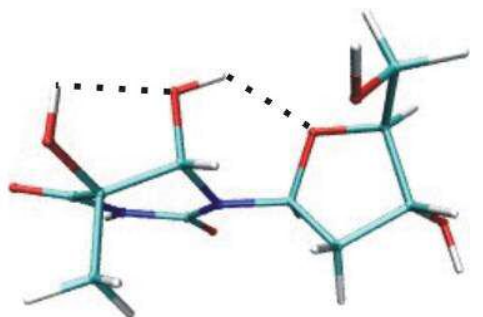

sTg3 
Figure 4. Purine nucleic acid bases with their 8-oxo-counterparts.

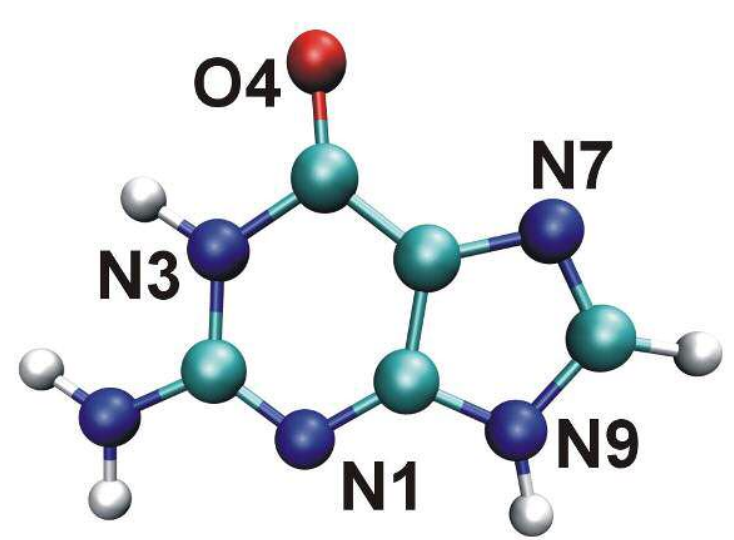

Guanine

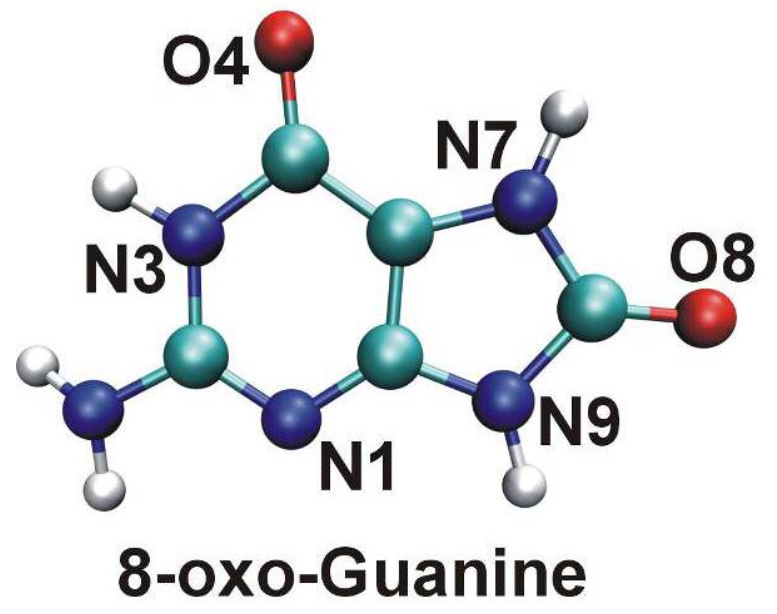

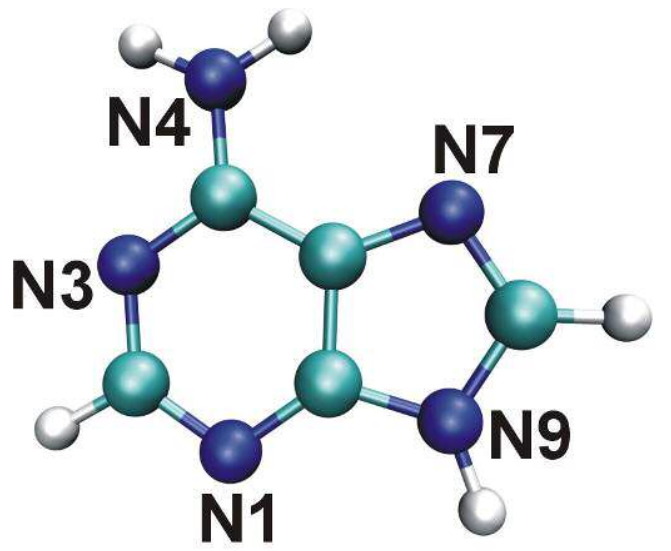

Adenine

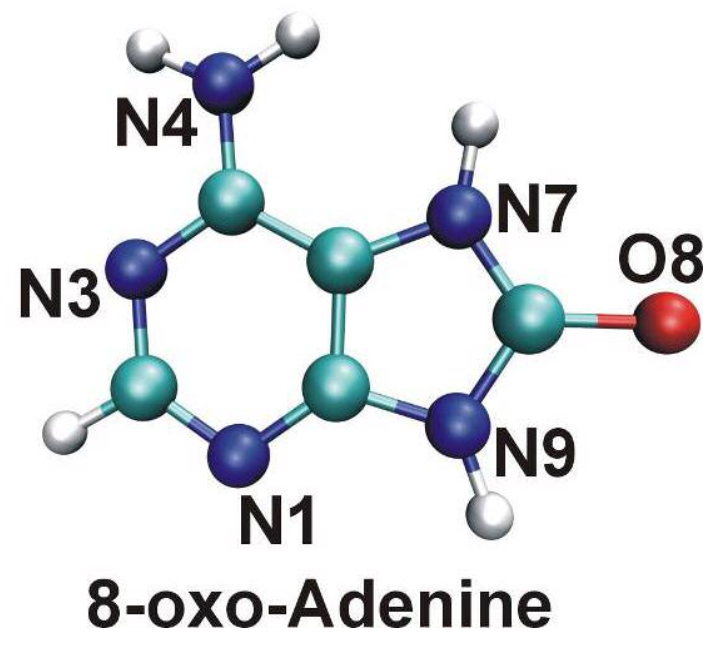


Figure 6. Points on the projection cylinder (A) and the molecular surface (B).

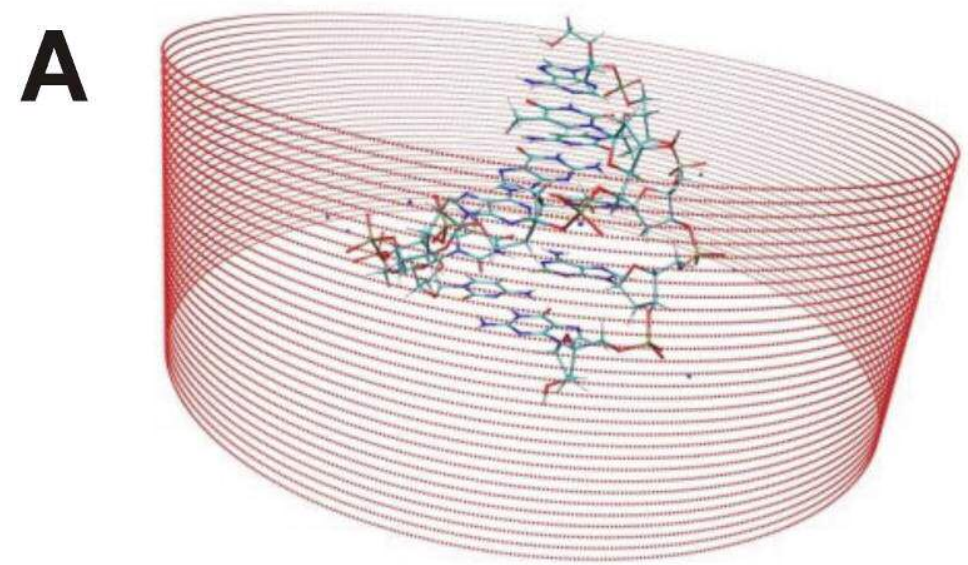

B

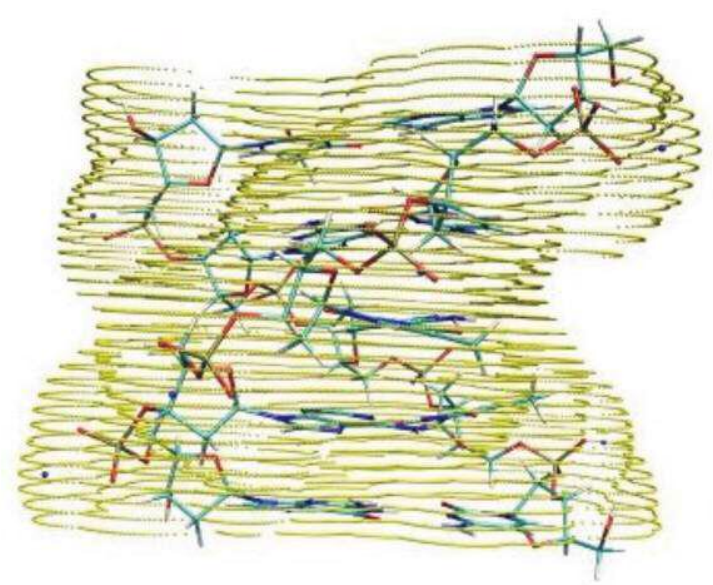


Figure 9. Two views of the superimposed intact (grey) and damaged (red) DNA pentamers show differences in geometry. The largest changes are marked with arrows.

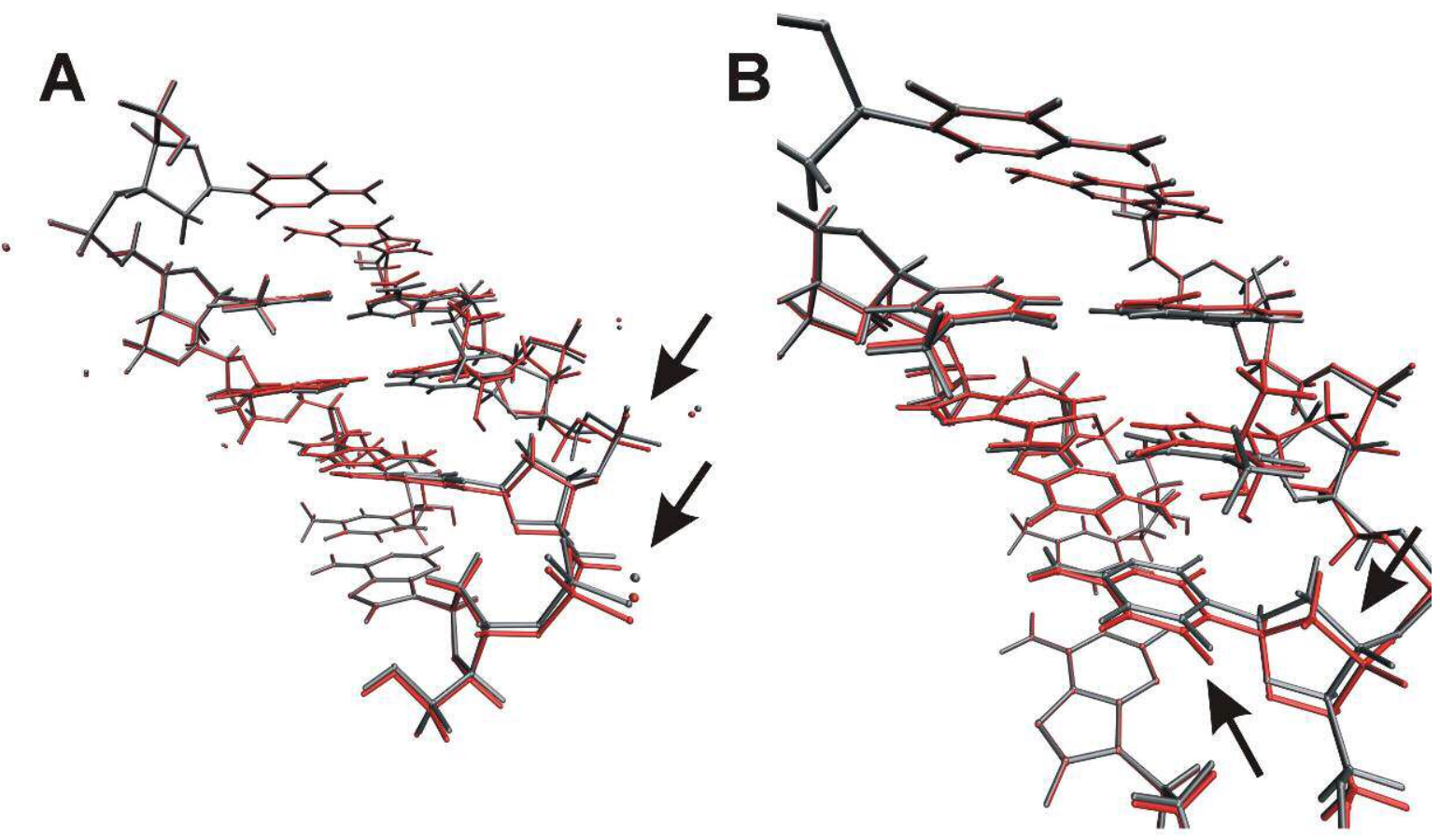


Supporting information

Figure S-1. Masks (black ellipses) used to extract information on positions of counteractions (labeled 1-8).

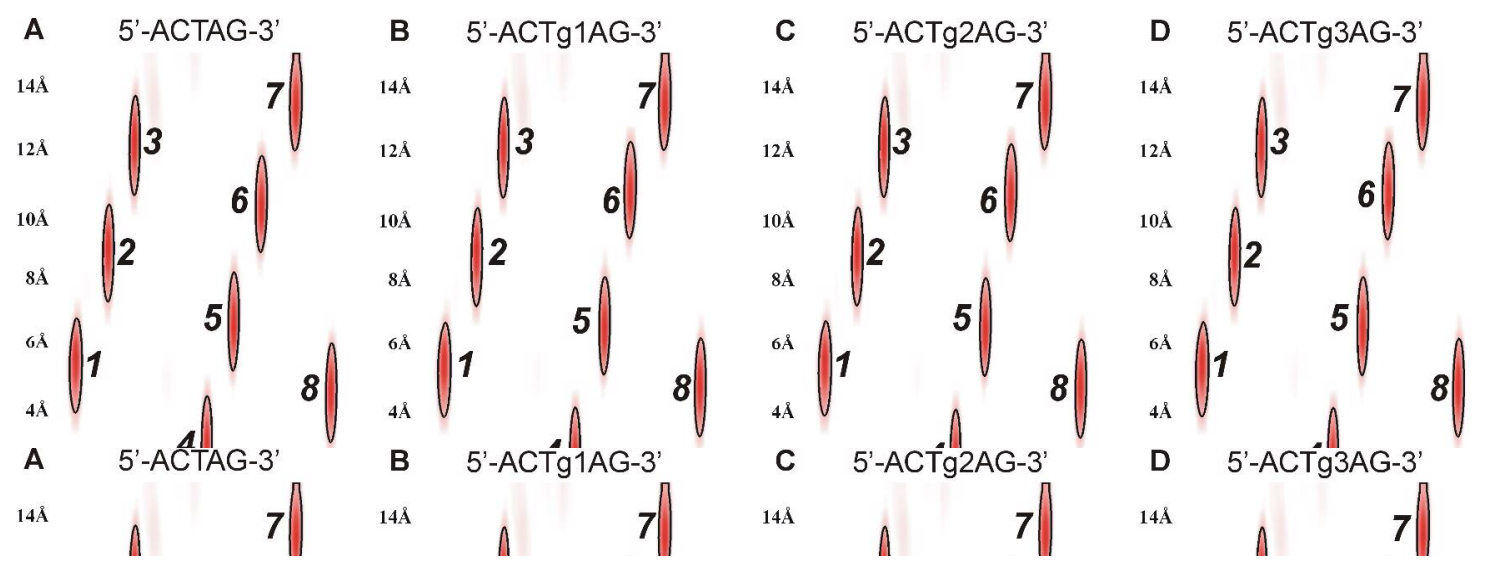

Figure S-2. Masks (irregular black curves) limiting the a and $\mathbf{b}$ features on EP maps.

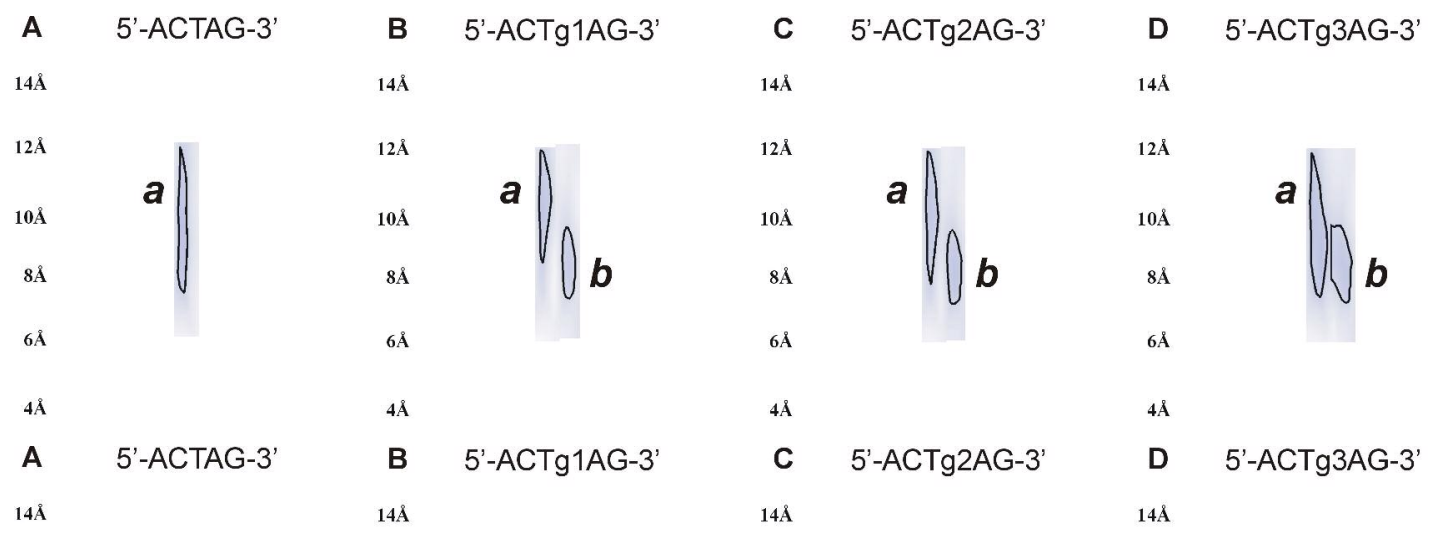


Figure S-3. Differences in geometry of DNA pentamers containing Tg1-Tg3: 5'ACTg1AG-3' (red), 5'-ACTg2AG-3' (green), 5'-ACTg1AG-3' (black).

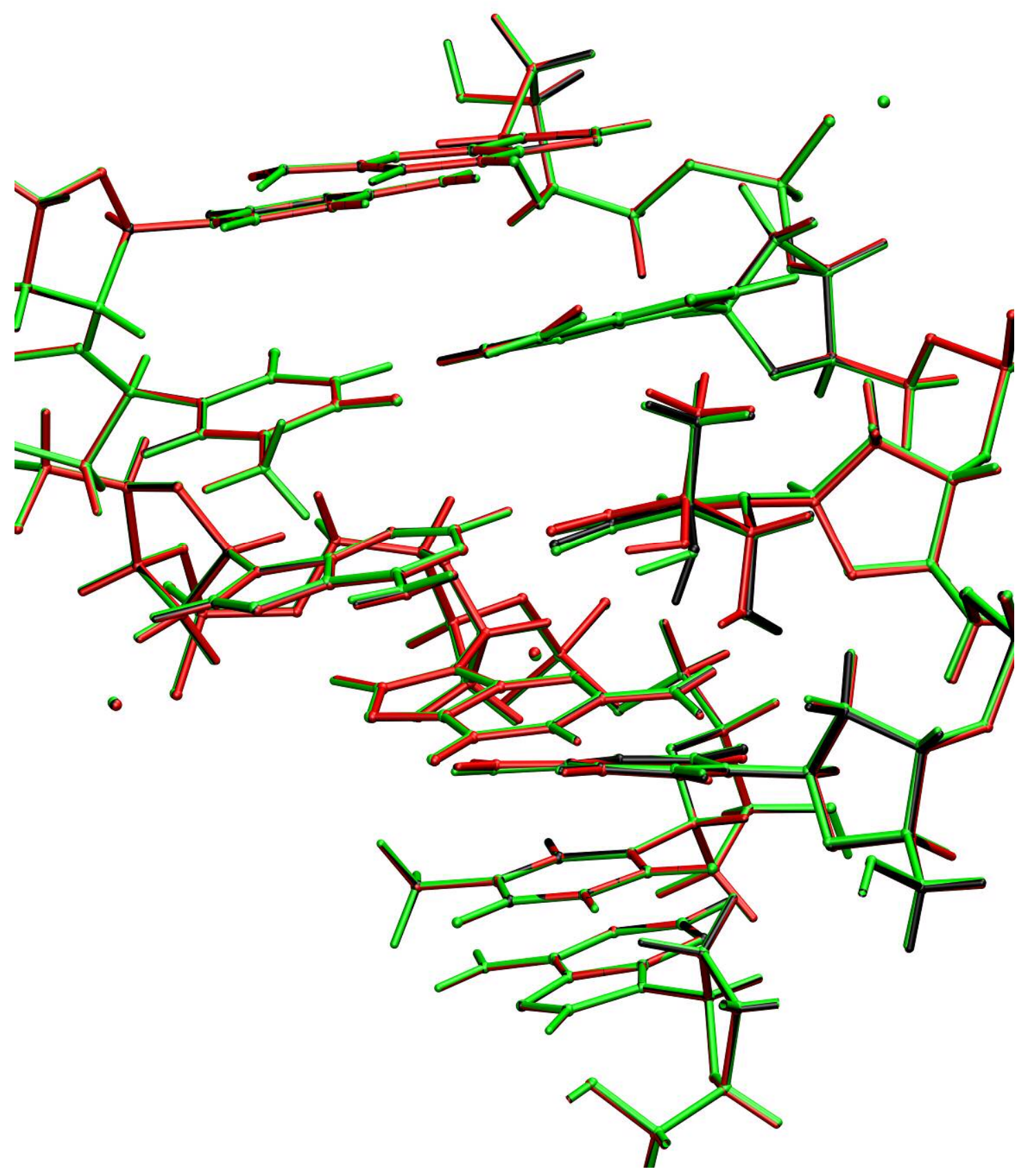

